\title{
Inhibition of the translocation and extracellular release of high-mobility group box 1 alleviates liver damage in fibrotic mice in response to D-galactosamine/lipopolysaccharide challenge
}

\author{
LI BAI ${ }^{1}$, MING KONG ${ }^{1}$, QINGFEN ZHENG ${ }^{1}$, XIAOHUI ZHANG ${ }^{1}$, XIN LIU $^{2}$, KEJIA ZU $^{3}$, YU CHEN $^{1}$, \\ SUJUN ZHENG ${ }^{1}$, JUNFENG LI ${ }^{1}$, FENG REN ${ }^{4}$, JINLI LOU ${ }^{2}$, SHUANG LIU ${ }^{1}$ and ZHONGPING DUAN ${ }^{1}$ \\ ${ }^{1}$ Artificial Liver Center; ${ }^{2}$ Clinical Laboratory Center; ${ }^{3}$ Department of Pathology, Beijing YouAn Hospital, \\ Capital Medical University; ${ }^{4}$ Research Department, Beijing Institute of Liver Diseases, Beijing 100069, P.R. China
}

Received February 22, 2015; Accepted December 8, 2015

DOI: $10.3892 / \mathrm{mmr} .2016 .5003$

\begin{abstract}
Acute liver injury in the setting of fibrosis is an area of interest in investigations, and remains to be fully elucidated. Previous studies have suggested the beneficial effects of liver fibrosis induced by thioacetamide and partial bile duct ligation against Fas-mediated acute liver injury. The activation of AKT and extracellular signal-regulated kinase signaling is considered to be crucial in this hepatoprotection. To demonstrate the protection of $\mathrm{CCl}_{4}$-induced liver fibrosis against lethal challenge, the present study compared the reactivity to lethal doses of D-galactosamine (D-GalN)/lipopolysaccharide (LPS) between fibrotic mice and control mice groups. The extent of hepatic damage was assessed by survival rate and histopathological analysis. The molecular basis of the fibrosis-based hepatoprotection was examined, with a particular focus on the translocation and release of high-mobility group box (HMGB)1 and the inflammatory response triggered by HMGB1. Hepatoprotection induced by fibrosis was demonstrated by improved survival rates $(100 \%$, vs. $20 \%)$ and improved preservation of liver architecture in fibrotic mice subjected to D-GalN/LPS, compared with control mice treated in the same way. D-GalN/LPS evoked the translocation and release of HMGB1, detected by immunohistochemistry, in the control mice, which was significantly inhibited in the fibrotic mice. The gene expression levels of HMGB1-associated proinflammatory cytokines, including interleukin (IL)-1 $\beta$, IL-6, tumor necrosis factor- $\alpha$ and IL-12p40, were markedly inhibited in the fibrotic mice when exposed to D-GalN/LPS. These findings confirmed that $\mathrm{CCl}_{4}$-based fibrosis induced hepatoprotection, and provided evidence that fibrosis inhibited
\end{abstract}

Correspondence to: Dr Zhongping Duan, Artificial Liver Center, Beijing YouAn Hospital, Capital Medical University, 8 Xitoutiao, YouAn Men Wai Avenue, Beijing 100069, P.R. China

E-mail: duan2517@163.com

Key words: liver fibrosis, drug-induced acute injury, hepatoprotection, high-mobility group box 1 the translocation and release of HMGB1, and the proinflammatory response triggered by HMGB1. This alleviated liver damage following exposure to D-GalN/LPS challenge.

\section{Introduction}

Liver fibrosis describes the excessive accumulation of extracellular matrix, which occurs in the majority of chronic liver diseases. Advanced fibrosis results in cirrhosis, portal hypertension and liver failure, and often requires liver transplantation (1-3). From another perspective, liver fibrosis represents the wound-healing response to chronic injury, which is a physiological response when tissues are "under attack' (3-5). When the tissue injury is severe or repetitive, or the wound-healing response itself becomes dysregulated, normal tissue repair can evolve into a progressively irreversible fibrotic response. Under these circumstances, excessive collagen deposition in and around the inflamed or damaged tissue distorts normal tissue architecture, leading to hepatocellular dysfunction and increased hepatic resistance to blood flow This causes hepatic insufficiency, portal hypertension and the eventual succumbing to mortality, as observed in end-stage liver disease $(2,3,5)$.

There has been encouraging evidence that fibrosis exerts pivotal, but divergent, effects on the liver. Although it is widely accepted that hepatic fibrosis results in deleterious effects, as described above, increasing evidence suggests a more favorable effect of liver fibrosis. In a mouse model of partial bile duct ligation (PBDL), injured ligated lobes exhibit improved tolerance to tumor necrosis factor (TNF)- $\alpha$ - and Fas-induced hepatocyte apoptosis, compared with non-ligated lobes, preventing mass hemorrhage and protecting mice from liver failure (6). In accordance with this, in vitro and in vivo animal experiments, performed by Bourbonnais et al, indicated that hepatocytes exposed to type I collagen, or fibrotic mice induced with thioacetamide (TAA), were less vulnerable to injury (7). However, the association between liver fibrosis induced by $\mathrm{CCl}_{4}$ and subsequent acute injury remains to be elucidated.

High-mobility group box (HMGB)1 is an evolutionarily conserved protein, which is present in the nucleus of almost 
all eukaryotic cells (8). The function of HMGB1 is diverse and compartment-specific. As a DNA chaperone, nuclear HMGB1 is engaged in several DNA-activity-associated events, including DNA replication, recombination, transcription and repair. HMGB1 can be actively secreted by innate immune cells or passively released by dead, dying or injured cells. Extracellular HMGB1, as a damage-associated molecular pattern (DAMP), is critically involved in several pathophysiological processes, including infection, tissue injury, inflammation, apoptosis and the immune response (9-11). All these characteristics make HMGB1 a critical molecular target in several diseases, including infection, ischemia-reperfusion injury, immune disorders and cancer (11-16).

The profibrotic function of HMGB1 has been demonstrated previously $(9,10,17,18)$. The upregulation of HMGB1 during liver fibrosis may be involved in tissue remodeling and fibrogenesis through the direct activation of hepatic stellate cells (HSCs). Therefore, inhibiting the bioavailability of HMGB1 may constitute a therapeutic strategy for the treatment of liver fibrosis $(17,18)$. Accumulating evidence has indicated that HMGB1 is critical in the pathogenesis of acute liver injury/failure originating from a variety of stimuli (19-22). HMGB1 has been reported as a sensitive serum diagnostic and biomarker for the assessment of severity in patients with acute liver injury $(22,23)$.

To support the hypothesis, the translocation and extracellular release of HMGB1 were compared between control and fibrotic mice in response to D-GalN/LPS challenge, and inflammatory response mediated by HMGB1 was analyzed. The present study aims to demonstrate that hepatoprotection induced by liver fibrosis is mediated by HMGB1. These findings will provide novel interpretation for the pathogenesis of acute liver injury, in the setting of hepatic fibrosis. This may, at least in part, account for the reduced inflammatory response and alleviation of liver damage in these mice.

\section{Materials and methods}

Animals. A total of 35 male Balb/c mice (6-8-week-old; 20-25 g) were purchased from Laboratory Animal Center, Academy of Military Medical Sciences, Beijing, China. The animals were housed in a specific pathogen-free environment under controlled conditions $\left(22-24^{\circ} \mathrm{C}, 12-\mathrm{h}\right.$ light/dark cycle) and fed an AIN93 diet with access to water throughout the experiment. Experimental procedures were approved by the Institutional Animal Care and Use Committee at Beijing YouAn Hospital affiliated to Capital Medical University (Beijing, China), according to the Guide for the Care and Use of Laboratory Animals (24).

Experimental designs. To investigate the correlation between liver fibrosis and injury tolerance, the following animal models were developed: i) Induction of fibrosis: liver fibrosis was established by intraperitoneal injection of $\mathrm{CCl}_{4}$ (Sinopharm Chemical Reagent Beijing Co., Ltd., Beijing, China) in mineral oil (Amresco LLC, Solon, OH, USA) twice weekly, for 6 weeks. The initial dose of $\mathrm{CCl}_{4}$ was $0.2 \mu \mathrm{l} / \mathrm{g}$, following which the doses increased gradually, up to $3 \mu \mathrm{l} / \mathrm{g}$ ii) Acute challenge: Control and fibrotic mice were sacrificed by a lethal dose of hepatic toxins ( $1 \mathrm{mg} / \mathrm{g}$ D-GalN+50 ng/g LPS; Sigma-Aldrich,
St. Louis, MO, USA). The mice were divided as follows: Control 5, D-GalN/LPS 10, fibrosis 10, and Fib+ D-GalN/LPS 10. Sera and liver tissues were harvested from the mice at indicated time points for analysis. Following harvesting, a section of the liver was fixed in 10\% neutral-buffered formalin (Sinopharm Chemical Reagent Beijing Co., Ltd.) for histological analysis and immunostaining. The remaining liver was cut into pieces and snap-frozen for homogenization to extract total liver RNA.

Evaluation of liver injury. The liver tissues fixed in $10 \%$ formalin were embedded in paraffin (Sinopharm Chemical Reagent Beijing Co., Ltd.), sectioned into $3 \mu \mathrm{m}$ sections and stained with hematoxylin and eosin (Sinopharm Chemical Reagent Beijing Co., Ltd.) for light microscopy. Histological severity of liver injury was graded numerically, according to the pathological grading criteria described by Lefkowitch (25). The parameters were graded with a score between 0 and 6 , with 0 indicating no abnormality, 1 or 2 indicating mild liver injury, 3 or 4 moderate injury, and 5 or 6 severe injury.

SYBR Green reverse-transcription-quantitative polymerase chain reaction. Frozen liver tissue $(\sim 50 \mathrm{mg})$ was cut into pieces, and homogenized in $1 \mathrm{ml}$ of TRIzol reagent (Invitrogen; Thermo Fisher Scientific, Inc., Waltham, MA, USA) using an electric homogenizer (Tissue Tearor, BioSpec Products Inc., Bartlesville, OK, USA) on ice. Total RNA was extracted according to the manufacturer's protocol. cDNA was synthesized from $2.5 \mu \mathrm{g}$ RNA using random primers and an AMV Retrotranscriptase system (Takara Biotechnology Co., Ltd., Dalian, China) using the following temperature protocol: $30^{\circ} \mathrm{C}$ for $10 \mathrm{~min} ; 42^{\circ} \mathrm{C}$ for $30 \mathrm{~min}$ and $95^{\circ} \mathrm{C}$ for $5 \mathrm{~min}$. The SYBR Green RT-qPCR was performed using the ABI StepOne Plus and software (Applied Biosystems; Thermo Fisher Scientific, Inc.). All reactions were performed in triplicate. In a final reaction volume of $20 \mu \mathrm{l}$, the following were added: $1 \mathrm{X}$ SYBR Green (Takara Biotechnology Co., Ltd.) cDNA, $0.5 \mathrm{mM}$ each primer and ROX (Takara Biotechnology Co., Ltd.). The conditions of the qPCR reaction were as follows: $50^{\circ} \mathrm{C}(2 \mathrm{~min})$, $95^{\circ} \mathrm{C}(5 \mathrm{~min})$, followed by 40 cycles of $95^{\circ} \mathrm{C}(15 \mathrm{sec})$ and $60^{\circ} \mathrm{C}(30 \mathrm{sec})$. The primers used were designed using Primer version 3.0 (26) and the sequences are listed in Table I. The relative expression levels of the target genes were calculated and normalized to the expression of GAPDH, a housekeeping gene.

Immunofluorescence. The liver tissues were snap-frozen in liquid nitrogen (Haotian Corporation, Beijing, China) and embedded in Tissue-Tek OCT (Sakura Finetek USA Inc., Torrance, CA, USA) compound. For immunofluorescence staining, the liver sections were fixed and stained with the following primary antibodies at $4^{\circ} \mathrm{C}$ overnight: Rabbit anti-mouse HMGB1 monoclonal antibody (1:100; cat. no ab79823, Abcam, Cambridge, MA, USA), goat anti-mouse collagen type I (1:200; cat. no. 1310-01, Southern Biotech, San Diego, CA, USA) and DAPI (EMD Millipore, Billerica, MA, USA). For indirect immunofluorescence staining, liver sections were incubated with the following secondary antibodies at $37^{\circ} \mathrm{C}$ for $30 \mathrm{~min}$ : fluorescein isothiocyanate-conjugated donkey anti-rabbit IgG for HMGB1 (1:500; cat. no. sc-2090; Santa Cruz Biotechnology, Inc., 
Table I. Primer sequences used for reverse transcription-quantitative polymerase chain reaction analysis.

\begin{tabular}{lll}
\hline Gene & \multicolumn{1}{c}{ Sense } & \multicolumn{1}{c}{ Anti-sense } \\
\hline GAPDH & 5'-AACTTTGGCATTGTGGAAGG-3' & 5'-ACACATTGGGGGTAGGAACA-3' \\
IL-1 $\beta$ & 5'-GCCCATCCTCTGTGACTCAT-3' & 5'-AGGCCACAGGTATTTTGT-3' \\
IL-6 & 5'-AGTTGCCTTCTTGGGACTGA-3' & 5'-TCCACGATTTCCCAGAGAAC-3' \\
IL-12p40 & 5'-CAGCTTCTTCATCAGGGACAT-3' & 5'-CTTGAGGGAGAAGTAGGAATGG-3' \\
TNF- $\alpha$ & 5'-GCCTCTTCTCATTCCTGCTTGT-3' & 5'-TTGAGATCCATGCCGTTG-3
\end{tabular}

IL, interleukin; TNF, tumor necrosis factor.

Dallas, TX, USA) and Cy3-conjugated rabbit anti-goat IgG for collagen type I (1:500; cat. no. C2821, Sigma-Aldrich) were used. The results were visualised and quantified using a Inverted Flourescence Microscope ECLIPSE Ti and NIS-Elements F 3.0 software (Nikon Corporation, Tokyo, Japan)

HMGB1 immunohistochemical staining. Following deparaffinization and rehydration, the embedded liver sections were treated with $3 \% \mathrm{H}_{2} \mathrm{O}_{2}$ for $15 \mathrm{~min}$, followed by microwave antigen retrieval for a further $15 \mathrm{~min}$ in citrate buffer (Sinopharm Chemical Reagent Beijing Co., Ltd.). The nonspecific proteins were blocked with $10 \%$ goat serum for $30 \mathrm{~min}$. For HMGB1 staining, the specimens were incubated with rabbit anti-mouse HMGB1 monoclonal antibody (cat. no. ab79823; 1:200; Abcam) overnight at $4^{\circ} \mathrm{C}$, followed by 30 min incubation with horseradish-peroxidase-conjugated goat anti-rabbit secondary antibody (cat. no. ZB-2301; 1:200; Zhongshan Golden Bridge Biotechnology Co,. Ltd., Beijing, China). The sections were incubated with diaminobenzidine (Zhongshan Golden Bridge Biotechnology Co., Ltd.) for $5 \mathrm{~min}$ as a chromogenic substrate and were counterstained with hematoxylin. The tissue sections were then dehydrated and stabilized with mounting medium (Zhongshan Golden Bridge Biotechnology Co., Ltd.). Images were captured using Bx51 microscope (Olympus America, Inc., Melville, NY, USA) and cellSens software (version 1.4.1.; Olympus Corporation, Tokyo, Japan)

Statistical analysis. Graphpad Prism version 5.0 (GraphPad Software, San Diego, CA, USA) was used for data processing and analysis. Results are expressed as the mean \pm standard error of the mean. Group comparisons were performed using one-way analysis of variance or Student's t-test. $\mathrm{P}<0.05$ was considered to indicate a statistically significant difference.

\section{Results}

Survival of fibrotic mice following exposure to a lethal dose of $D$-GalN/LPS. The present study examined the effect of fibrosis on the survival rates of mice in response to hepatic toxins. Control and fibrotic mice were subjected to lethal doses of D-GalN (1 $\mu \mathrm{g} / \mathrm{g})$ plus LPS (50 $\mathrm{ng} / \mathrm{g})$ as an acute insult. During $24 \mathrm{~h}$ observation, $8 / 10$ of the mice died in the control group treated with D-GalN/LPS (20\% survival rate), however, no fibrotic mice succumbed to mortality when exposed to the same dose of D-GalN/LPS (survival rate 100\%; Fig. 1). This

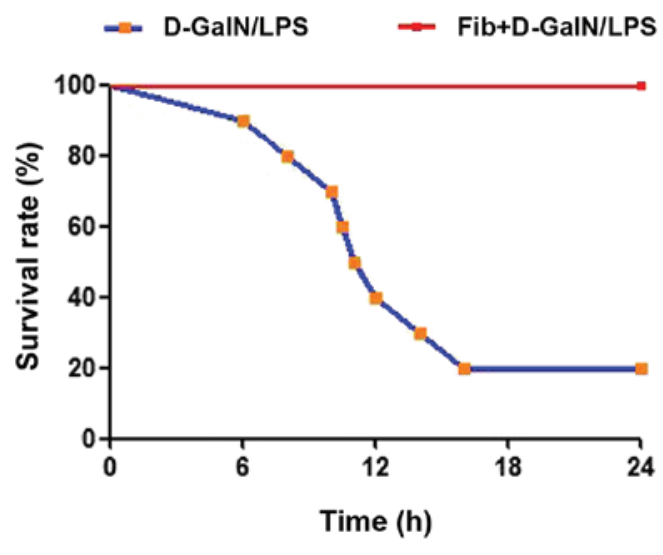

Figure 1. Fibrotic mice $(n=10)$ survive lethal doses of D-GalN/LPS. The mice in the control and fibrosis groups were subjected to lethal doses of D-GalN $(1 \mu \mathrm{g} / \mathrm{g})+\mathrm{LPS}(50 \mathrm{ng} / \mathrm{g})$ as an acute insult. The survival rate during $24 \mathrm{~h}$ was observed. D-GaIN, D-galactosamine; LPS, lipopolysaccharide.

provided direct and macroscopic evidence of the advantageous effect of fibrosis. The extent of hepatic damage was analyzed and compared between the control and fibrotic mice treated with D-GalN/LPS. Microscopic examination of liver tissues from control mice treated with D-GalN/LPS revealed pronounced hepatocyte destruction with mass hemorrhage. By contrast, marked improvements in histology were noted in the $\mathrm{CCl}_{4}$-induced fibrotic mice treated in the same way (Fig. 2A). Histological grading provided further quantitative information of the extent of liver damage, and the difference between the control and the fibrotic mice in response to D-GalN/LPS was significant $(\mathrm{P}=0.0353$; Fig. $2 \mathrm{~B})$. Improved survival rates and preservation of liver architecture provided compelling evidence that fibrosis may result in decreased susceptibility of hepatocytes to lethal doses of D-GalN/LPS.

Translocation and extracellular release of $H M G B 1$ is inhibited in fibrotic mice treated with D-GalN/LPS. The present study evaluated the immunoreactive expression of HMGB1, which is important in the initiation and progression of proinflammatory processes (12). As the functions of HMGB1 are diverse and compartment-specific (9-11), the cellular localization of HMGB1 in the liver was determined in the present study. The liver tissues were obtained from control mice (at any time point), control mice upon D-GalN/LPS challenge (at the time of death), fibrotic mice ( $24 \mathrm{~h}$ after the last $\mathrm{CCl}_{4}$ injection), and fibrotic mice upon D-GalN/LPS challenge 
A
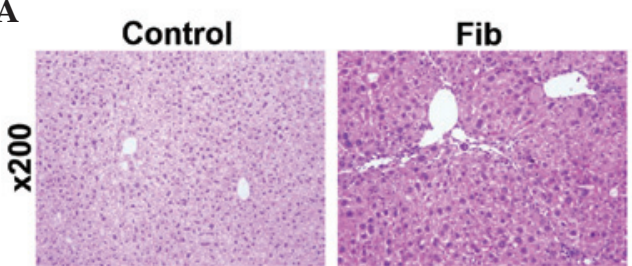

D-GaIN/LPS
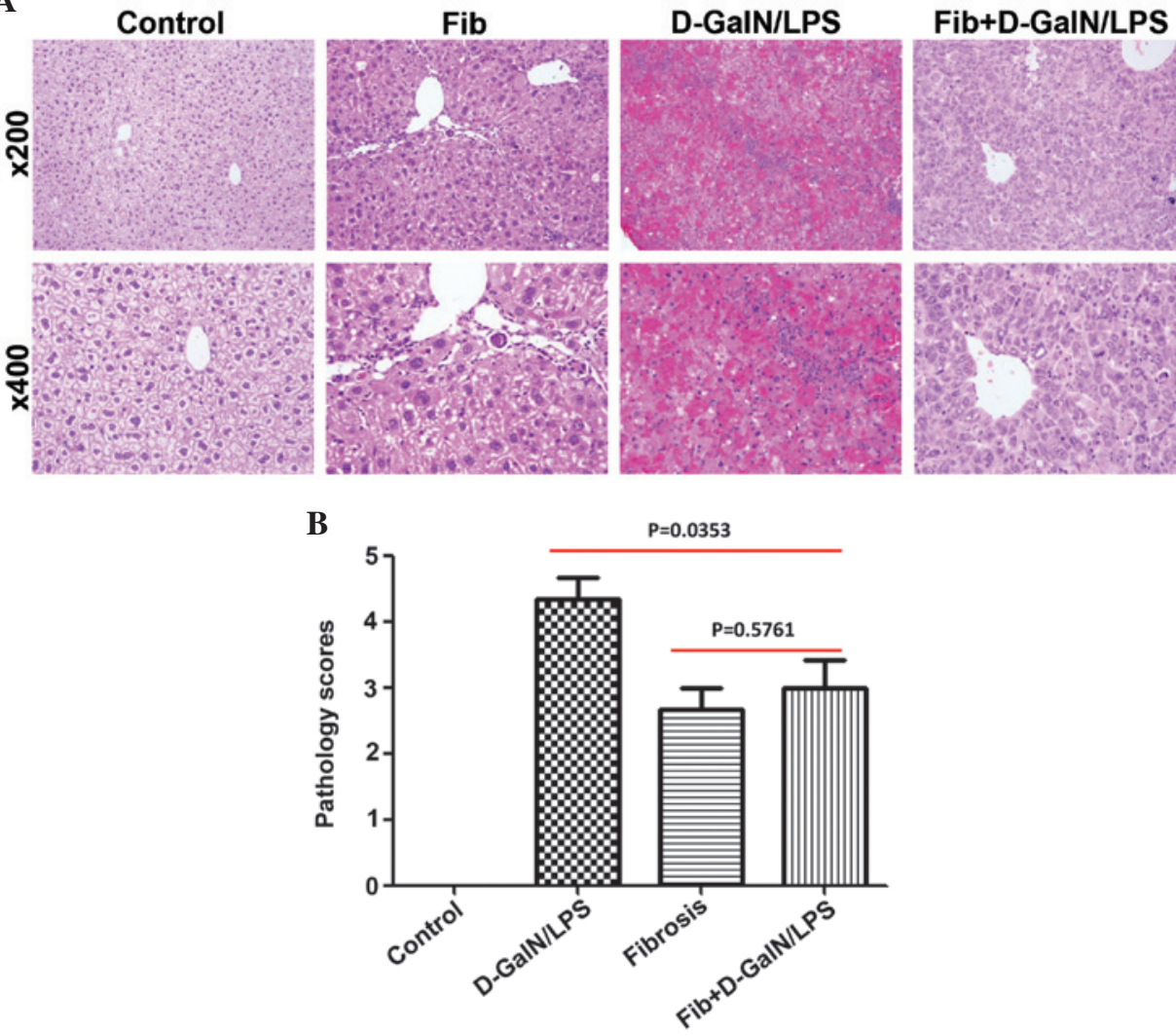

Figure 2. Histopathological characteristics are markedly improved in fibrotic mice exposed to D-GalN/LPS. (A) Representative images of liver histology in all groups, determined using hematoxylin and eosin staining. (Original magnification, upper panels, x200; lower panels, x400). (B) Average pathology scores in the different groups. Histological severity of liver injury was assessed by pathologists blindly. The scores were compared between the different groups. Data are presented as the mean \pm standard error of the mean. D-GaIN, D-galactosamine; LPS, lipopolysaccharide; Fib, fibrosis.
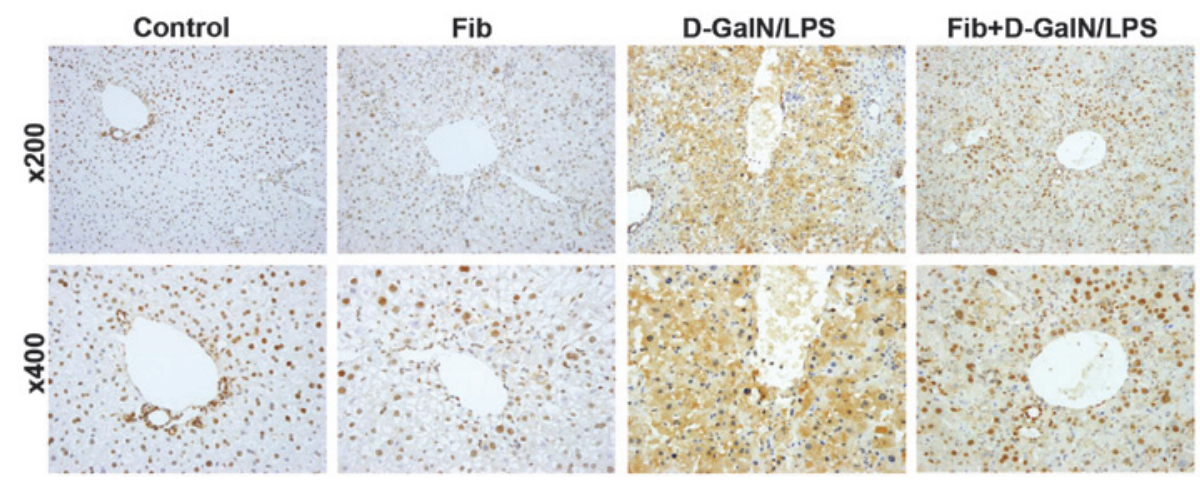

Figure 3. Translocation and extracellular release of HMGB1 triggered by D-GalN/LPS are significantly inhibited in fibrotic mice. Expression of HMGB1 was detected using immunohistochemistry in control and fibrotic mice treated with or without D-GalN/LPS. Representative HMGB1 staining of all groups is shown. (Original magnification, upper panels, x200; lower panels, x400). HMGB1, high-mobility group box 1; D-GaIN, D-galactosamine; LPS, lipopolysaccharide; Fib, fibrosis.

(24 h after D-GalN/LPS challenge). Immunohistochemical staining with antibodies against HMGB1 revealed a distinct expression pattern in paraffin sections retrieved from control and fibrotic mice, with or without D-GalN/LPS challenge. HMGB1 was localized in the nucleus of the majority of the hepatocytes in the control mice. In the fibrotic liver tissues, a relative increase in the expression of HMGB1 was observed, and extranuclear HMGB1-positive staining was visible in a number of hepatocytes. Following D-GalN/LPS challenge, HMGB1-positive staining was significantly enhanced, however, immunoreactivity for HMGB1 in the nucleus was markedly reduced, and translocation and aberrant extracellular expression of HMGB1 were observed. The expression of HMGB1 and its translocation into extranuclear and extracellular milieu were significantly inhibited in the fibrotic mice treated with the same dose of D-GalN/LPS (Fig. 3). The distribution of HMGB1 coincided with that of type I collagen in the fibrotic tissues on visualization following immunofluorescent staining, supporting the close correlation between fibrosis and the expression of HMGB1 (Fig. 4). 

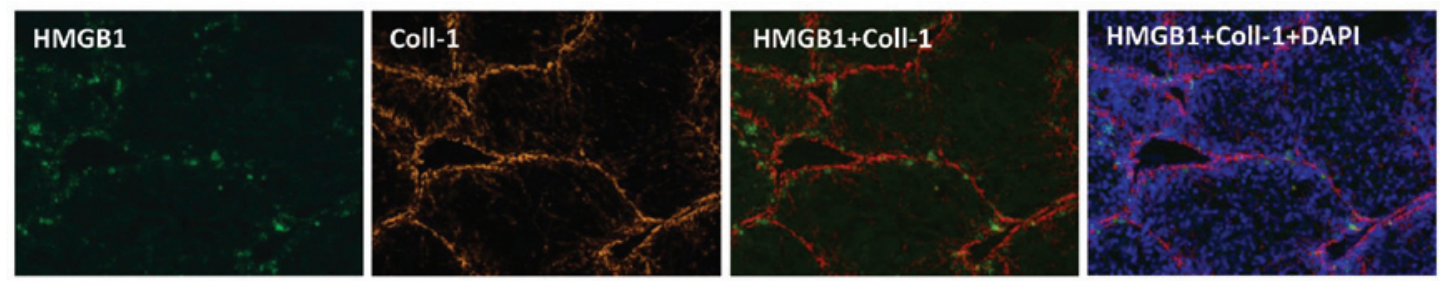

Figure 4. Distribution of HMGB1 is in accordance with that of type I collagen in fibrotic mice. Expression levels of HMGB1 were detected using immunofluorescence. Representative HMGB1 staining of all groups is shown (Original magnification, x200). HMGB1, high-mobility group box 1; Coll-1, type I collagen.
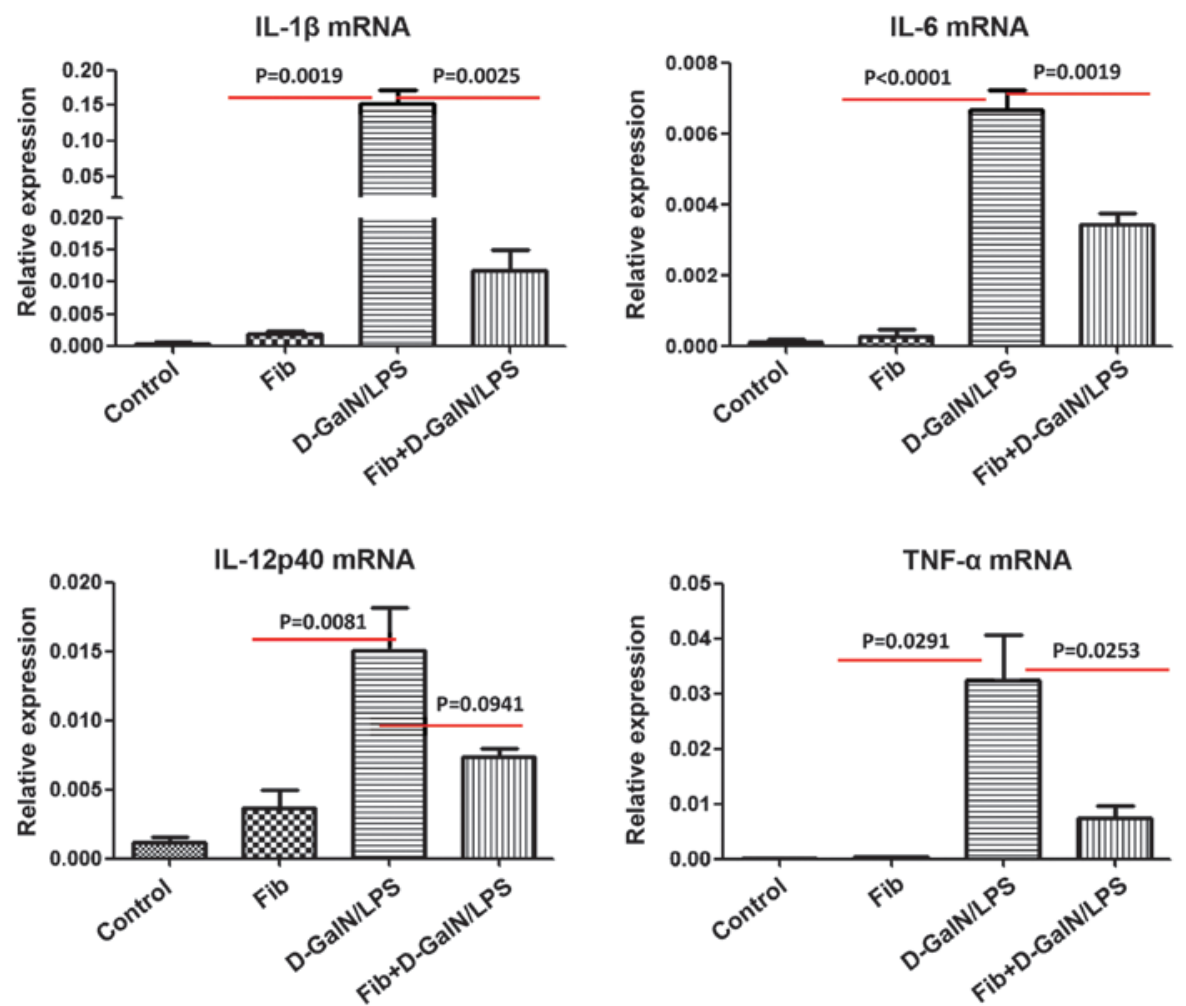

Figure 5. HMGB1-mediated inflammatory responses are inhibited in fibrotic mice treated with D-GalN/LPS. The mRNA expression levels of IL-1 $\beta$, IL-6, TNF- $\alpha$ and IL-12P40 inflammatory genes were determined using reverse transcription-quantitative polymerase chain reaction analysis. Data are presented as the mean \pm standard error of the mean. HMGB1, high-mobility group box 1; D-GaIN, D-galactosamine; LPS, lipopolysaccharide; Fib, fibrosis; IL, interleukin; $\mathrm{TNF}$, tumor necrosis factor.

Accordingly, fibrosis may inhibit the expression and release of HMGB1 triggered by D-GalN/LPS, leading to alleviated liver damage.

HMGB1-mediated inflammatory responses are reduced in fibrotic mice treated with D-GalN/LPS. Increasing evidence suggests that HMGB1 can selectively bind multiple receptors, for example in receptors for advanced glycation end-products (RAGE) and Toll-like receptors, to activate different types of liver cells, including macrophages, neutrophils, dendritic cells and $\mathrm{T}$ cells, to produce cytokines, including TNF- $\alpha$, IL-1 $\beta$, IL-6, IL-10 and IL-12 (12). To further examine the molecular and immunological mechanisms involved in the development of reduced liver injury in fibrotic mice treated with D-GalN/LPS, the present study analyzed the mRNA expression levels of these HMGB1-triggered proinflammatory cytokines using RT-qPCR. The mRNA levels of IL-1 $\beta$, IL-6, TNF- $\alpha$ and IL-12P40 were significantly increased in the liver tissues retrieved from the control mice, which were exposed to lethal doses of D-GalN/LPS. The upregulation of these inflammatory cytokines was suppressed in the liver tissues retrieved from the fibrotic mice, which were exposed to D-GalN/LPS (Fig. 5). These data provided additional evidence that the HMGB1-mediated inflammatory and immune responses triggered by D-GalN/LPS were inhibited by liver fibrosis.

\section{Discussion}

The present study provided the first evidence, to the best of our knowledge, that inhibiting the expression, translocation and release of HMGB1 at least partially alleviated hepatic injury in $\mathrm{CCl}_{4}$-induced fibrotic mice exposed to D-GalN/LPS. The present study is the first to link the translocation and release of HMGB1 with resistance to fibrosis-based acute injury.

The identification of fibrosis as a key event in the alleviation of liver damage in response to insult is not unprecedented. 
Resistance to acute liver damage induced by TNF- $\alpha$ or Fas has been demonstrated in PBDL- and TAA-based models of fibrosis $(6,7)$. However, it remains important to verify and analyze this association in additional fibrosis models with different insults. The present study investigated the effects of $\mathrm{CCl}_{4}$-induced liver fibrosis, another well-established animal model for liver fibrosis, on the secondary challenge of D-GalN/LPS, which is widely used as an inducer for acute hepatic injury/failure. The fibrosis induced by $\mathrm{CCl}_{4}$ conferred significant protection against lethal challenge with D-GalN/LPS, as demonstrated by improved survival rates and improved preservation of liver architecture. Previous clinical data confirmed the ongoing fibrosis and HSC activation in the progression of acute liver failure, supporting the hypothesis that fibrosis in acute liver failure may be a physiological, and possibly beneficial, response by the liver $(27,28)$.

The molecular basis for the hepatoprotective response induced by liver fibrosis has been discussed in previous reports. Much attention has been paid to the balance between the survival and apoptosis of hepatocytes. In this regard, enhanced cell survival and liver regeneration, along with attenuated hepatocyte apoptosis, mediated by the activation of AKT and extracellular signal-regulated kinase signaling, have been documented $(6,7)$. However, inflammation is a major component of the pathology of drug-induced liver injury and liver fibrosis, and advances in the biology of inflammation have revealed that specific cytokines are important and effective pathogenic mediators (12). For this reason, it is imperative to determine whether HMGB1, an important proinflammatory mediator, functions in acute hepatic injury occurring in the setting of fibrosis, and to investigate the underlying mechanism.

HMGB1, which is constitutively expressed in the nucleus of the majority of cells under basal conditions, functions as a structural co-factor that is critical for proper transcriptional regulation. In the past decade, the active secretion of extracellular HMGB1 by innate immune cells in response to pathogenic products, and its release by injured or dying cells, has been identified to occupy a central role in the pathogenesis of sterile and infectious inflammation (12). Mechanistically, extracellular HMGB1 binds to pattern recognition receptors, including Tolllike receptor 4 and RAGE, and acts as a DAMP molecule to activate intracellular signals, including nuclear factor $-\kappa \mathrm{B}$ and the mitogen-activated protein kinase pathway, which regulate the gene expression of various immune and inflammatory mediators, including TNF- $\alpha$, IL-1 $\beta$, IL-6, IL-10 and IL-12. In addition, HMGB1 functions as an immune adjuvant, to trigger the activation of immune cells, including T cells, dendritic cells and endothelial cells, and the secretion of HMGB1, which forms a positive feedback loop that potentially amplifies local inflammatory responses by enhancing the release of cytokines and chemokines $(11,12,29)$.

HMGB1 is translocated from the nucleus to the cytoplasm, and is subsequently released into the extracellular milieu, which has been noted under certain conditions, including ischemia and reperfusion injury, hepatitis $\mathrm{C}$ virus or hepatitis $\mathrm{B}$ viral infections, and drug-induced acute liver failure $(20,21,23,25,30)$. Although the profibrotic function of HMGB1 is established, the effect of liver fibrosis on the translocation and extracellular release of HMGB1 remains to be fully elucidated. In the present study, lethal doses of D-GalN/LPS triggered the translocation and excessive release of HMGB1, accompanied by a significant upregulation in the gene expression levels of proinflammatory IL-1 $\beta$, IL-6, TNF- $\alpha$ and IL-12P40. By contrast, the increase in HMGB1 release and proinflammatory gene expression were markedly inhibited in fibrotic mice exposed to D-GalN/LPS. In addition, the distribution of HMGB1 detected by immunofluorescence staining was in accordance with that of type I collagen, suggesting that fibrosis was closely associated with the expression of HMGB1. Thus, the present study hypothesized that fibrosis inhibits the translocation and release of HMGB1, and the inflammatory response triggered by HMGB1. In addition, the release and activity of HMGB1 were restored following the resolution of liver fibrosis, supporting the inhibitory action of hepatic fibrosis on HMGB1 translocation (unpublished data).

Depending on the inducing stimulus, the mechanism underlying the secretion and release of HMGB1 can vary. In response to exogenous pathogen-associated molecular patterns, for example endotoxin, or endogenous inflammatory stimuli, for example DAMP molecules, HMGB1 is modified by different post-transcriptional modifications, including acetylation and phosphorylation, which impedes its re-entry into the nucleus, with subsequent migration into the cytoplasm and release into the extracellular milieu $(31,32)$. In the present study, the liver fibrosis induced by $\mathrm{CCl}_{4}$ may have altered the function of HMGB1 through effects on its post-transcriptional modifications.

Although current data are only associative, $\mathrm{CCl}_{4}$-induced fibrosis may function as a critical event in the inhibition of HMGB1 release and the resulting alleviation of liver injury. Understanding the physiological and beneficial roles of fibrosis in the progression of acute liver injury may reveal novel opportunities for the treatment of liver failure, particularly of acute-on-chronic liver failure.

\section{Acknowledgements}

This study was funded by the National Science and Technology Key Project of China on 'Major Infectious Diseases such as HIV/AIDS, Viral Hepatitis Prevention and Treatment' (grant no. 2012ZX10002004-006, 2012ZX10004904-003-001 and 2013ZX10002002-006), the High Technical Personnel Training Item in Beijing Health System (grant nos. 2011-3-083 and 2013-3-071), the Special Fund for Clinical Medicine Development of Beijing Municipal Administration of Hospitals (grant no. XM201308), the National Key Subject Construction Project (grant nos. WJWYA-2014-002 and WJWYA-2014-004) and the Basic-Clinical Cooperation Project of Capital Medical University (grant no. 14JL72, 14JL73).

\section{References}

1. Friedman SL: Mechanisms of hepatic fibrogenesis. Gastroenterology 134: 1655-1669, 2008.

2. Friedman SL: Evolving challenges in hepatic fibrosis. Nat Rev Gastroenterol Hepatol 7: 425-436, 2010.

3. Bataller R and Brenner DA: Liver fibrosis. J Clin Invest 115: 209-218, 2005.

4. White ES and Mantovani AR: Inflammation, wound repair and fibrosis: Reassessing the spectrum of tissue injury and resolution. J Pathol 229: 141-144, 2013.

5. Wynn TA and Ramalingam TR: Mechanisms of fibrosis: Therapeutic translation for fibrotic disease. Nat Med 18: 1028-1040, 2012. 
6. Osawa Y, Hannun YA, Proia RL and Brenner DA: Roles of AKT and sphingosine kinase in the antiapoptotic effects of bile duct ligation in mouse liver. Hepatology 42: 1320-1328, 2005.

7. Bourbonnais E, Raymond VA, Ethier C, Nguyen BN, El-Leil MS, Meloche $\mathrm{S}$ and Bilodeau M: Liver fibrosis protects mice from acute hepatocellular injury. Gastroenterology 142: 130-139.e4, 2012.

8. Javaherian K, Liu JF and Wang JC: Nonhistone proteins HMG1 and HMG2 change the DNA helical structure. Science 199: 1345-1346, 1978

9. Chen R, Hou W, Zhang Q, Kang R, Fan XG and Tang D: Emerging role of high-mobility group box 1 (HMGB1) in liver diseases. Mol Med 19: 357-366, 2013.

10. Li LC, Gao J and Li J: Emerging role of HMGB1 in fibrotic diseases. J Cell Mol Med 18: 2331-2339, 2014.

11. Kang R, Chen R, Zhang Q, Hou W, Wu S, Cao L, Huang J, Yu Y, Fan XG, Yan Z, et al: HMGB1 in health and disease. Mol Aspects Med 40: 1-116, 2014.

12. Andersson U and Tracey KJ: HMGB1 is a therapeutic target for sterile inflammation and infection. Annu Rev Immunol 29 139-162, 2011.

13. Huang H, Nace GW, McDonald KA, Tai S, Klune JR, Rosborough BR, Ding Q, Loughran P, Zhu X, Beer-Stolz D, et al: Hepatocyte-specific high-mobility group box 1 deletion worsens the injury in liver ischemia/reperfusion: A role for intracellular high-mobility group box 1 in cellular protection. Hepatology 59: 1984-1997, 2014.

14. Kamo N, Ke B, Ghaffari AA, Shen XD, Busuttil RW, Cheng G and Kupiec-Weglinski JW: ASC/caspase-1/IL-1 $\beta$ signaling triggers inflammatory responses by promoting HMGB1 induction in liver ischemia/reperfusion injury. Hepatology 58: 351-362, 2013.

15. Sims GP, Rowe DC, Rietdijk ST, Herbst R and Coyle AJ: HMGB1 and RAGE in inflammation and cancer. Annu Rev Immunol 28 : 367-388, 2010

16. Yanai H, Ban T, Wang Z, Choi MK, Kawamura T, Negishi H, Nakasato M, Lu Y, Hangai S, Koshiba R, et al: HMGB proteins function as universal sentinels for nucleic-acid-mediated innate immune responses. Nature 462: 99-103, 2009.

17. Albayrak A, Uyanik MH, Cerrah S, Altas S, Dursun H, Demir M and Uslu H: Is HMGB1 a new indirect marker for revealing fibrosis in chronic hepatitis and a new therapeutic target in treatment? Viral Immunol 23: 633-638, 2010.

18. Wang FP, Li L, Li J, Wang JY, Wang LY and Jiang W: High mobility group box-1 promotes the proliferation and migration of hepatic stellate cells via TLR4-dependent signal pathways of PI3K/Akt and JNK. PLoS One 8: e64373, 2013.

19. Gong Q, Zhang H, Li JH, Duan LH, Zhong S, Kong XL, Zheng F, Tan Z, Xiong P, Chen G, et al: High-mobility group box 1 exacerbates concanavalin A-induced hepatic injury in mice. J Mol Med (Berl) 88: 1289-1298, 2010.
20. Zhou RR, Zhao SS, Zou MX, Zhang P, Zhang BX, Dai XH, Li N, Liu HB, Wang H and Fan XG: HMGB1 cytoplasmic translocation in patients with acute liver failure. BMC Gastroenterol 11: 21,2011

21. Kuroda N, Inoue K, Ikeda T, Hara Y, Wake K and Sato T: Apoptotic response through a high mobility box 1 protein-dependent mechanism in LPS/GalN-induced mouse liver failure and glycyrrhizin-mediated inhibition. PLoS One 9: e92884, 2014.

22. Antoine DJ, Jenkins RE, Dear JW, Williams DP, McGill MR, Sharpe MR, Craig DG, Simpson KJ, Jaeschke H and Park BK: Molecular forms of HMGB1 and keratin-18 as mechanistic biomarkers for mode of cell death and prognosis during clinical acetaminophen hepatotoxicity. J Hepatol 56: 1070-1079, 2012.

23. Tsung A, Klune JR, Zhang X, Jeyabalan G, Cao Z, Peng X, Stolz DB, Geller DA, Rosengart MR and Billiar TR: HMGB1 release induced by liver ischemia involves toll-like receptor 4 dependent reactive oxygen species production and calcium-mediated signaling. J Exp Med 204: 2913-2923, 2007.

24. National Research Council (US) Committee for the Update of the Guide for the Care and Use of Laboratory Animals: Guide for the Care and Use of Laboratory Animals. 8th edition. National Academies Press, Washington (DC), 2011.

25. Lefkowitch JH: Pathologic diagnosis of liver disease. In: Hepatology - A Textbook of Liver Disease. Zakim D and Boyer TD (eds). W.B. Saunders Company, Philadelphia, pp844-871, 1996.

26. Untergrasser A, Cutcutache I, Koressaar T, Ye J, Faircloth BC, Remm $M$ and Rozen SG: Primer 3 - new capabilities and interfaces. Nucleic Acids Res 40: e115, 2012.

27. Dechêne A, Sowa JP, Gieseler RK, Jochum C, Bechmann LP, El Fouly A, Schlattjan M, Saner F, Baba HA, Paul A, et al: Acute liver failure is associated with elevated liver stiffness and hepatic stellate cell activation. Hepatology 52: 1008-1016, 2010.

28. He Y, Jin L, Wang J, Yan Z, Chen T and Zhao Y: Mechanisms of fibrosis in acute liver failure. Liver Int 35: 1877-1885, 2015.

29. Yang H, Hreggvidsdottir HS, Palmblad K, Wang H, Ochani M, Li J, Lu B, Chavan S, Rosas-Ballina M, Al-Abed Y, et al: A critical cysteine is required for HMGB1 binding to Toll-like receptor 4 and activation of macrophage cytokine release. Proc Natl Acad Sci USA 107: 11942-11947, 2010.

30. Jung JH, Park JH, Jee MH, Keum SJ, Cho MS, Yoon SK and Jang SK: Hepatitis $C$ virus infection is blocked by HMGB1 released from virus-infected cells. J Virol 85: 9359-9368, 2011.

31. Youn JH and Shin JS: Nucleocytoplasmic shuttling of HMGB1 is regulated by phosphorylation that redirects it toward secretion. J Immunol 177: 7889-7897, 2006.

32. Bonaldi T, Talamo F, Scaffidi P, Ferrera D, Porto A, Bachi A, Rubartelli A, Agresti A and Bianchi ME: Monocytic cells hyperacetylate chromatin protein HMGB1 to redirect it towards secretion. EMBO J 22: 5551-5560, 2003. 